\title{
Endoscopic band ligation for colonic diverticular bleeding: possibility of standardization
}

Authors

Institution
Yuto Shimamura, Naoki Ishii, Fumio Omata, Noriatsu Imamura, Takeshi Okamoto, Mai Ego, Kaoru Nakano, Takashi Ikeya, Kenji Nakamura, Koichi Takagi, Katsuyuki Fukuda, Yoshiyuki Fujita

Department of Gastroenterology, St. Luke’s International Hospital, Tokyo, Japan submitted $\quad 8$. June 2015 accepted after revision 30. November 2015

\section{Bibliography}

Dol http://dx.doi.org/ 10.1055/s-0041-110954 Published online: 15.1.2016 Endoscopy International Open 2016; 04: E233-E237

(c) Georg Thieme Verlag KG Stuttgart · New York E-ISSN 2196-9736

\section{Corresponding author}

Yuto Shimamura, MD

Department of

Gastroenterology

St. Luke's International Hospital 9-1 Akashi-cho

Chuo-ku

Tokyo 104-0044

Japan

Fax: +81-03-3541-5151

yutshi@luke.ac.jp
Background and aims: Endoscopic band ligation (EBL) has been used to achieve hemostasis in patients with colonic diverticular bleeding. The safety and effectiveness of EBL when performed by non-expert endoscopists have not been sufficiently verified. This study aimed to elucidate the feasibility of the EBL technique when performed by non-expert endoscopists and of considering EBL as a standard treatment for colonic diverticular bleeding.

Patients and methods: A retrospective cohort study was conducted in a tertiary referral center in Tokyo, Japan, between June 2009 and October 2014. A total of 95 patients treated with EBL were included in the study and were divided into two groups according to whether they had been treated by expert or non-expert endoscopists. Comorbidities, medications, shock index, hemoglobin level on admission, location of the bleeding diverticula, rate of bowel preparation, procedure time, and EBL-associated adverse events were

\section{Introduction \\ $\nabla$}

Colonic diverticular bleeding is a major form of lower gastrointestinal bleeding [1-3]. Although the bleeding stops spontaneously in most cases, endoscopic, radiologic, or surgical treatment may be required if it persists $[4,5]$. When the source of bleeding is identified by colonoscopy, endoscopic hemostasis can be performed to prevent recurrent bleeding and decrease the need for surgery [6]. Several endoscopic hemostatic treatments for colonic diverticular bleeding are available, including endoscopic clipping, endoscopic band ligation (EBL), epinephrine injection, and contact thermal therapy [6-19]. However, they vary in effectiveness, and there is still no standardized therapy for this condition. Because of available local expertise, our unit prefers EBL as first-line therapy. Although endoscopic clipping is considered one of the most effective treatments, the di- evaluated in each group. Multivariate linear regression analyses were used to investigate factors related to EBL procedure time, which is the time elapsed between marking the site of bleeding with hemoclips and completion of the band release.

Results: A total of 47 (49.5\%) procedures were performed by expert endoscopists. In a bivariate analysis, the median EBL procedure times in the expert and non-expert groups were 15 minutes (range 4-45) and 11 minutes (range 4-36), respectively $(P=0.03)$. When a multivariate linear regression model was used, EBL for right-sided diverticula was the factor most significantly affecting EBL procedure time. No adverse events were encountered.

Conclusion: EBL can be safely and effectively performed by non-expert endoscopists. A right-sided location of diverticula was the factor most significantly affecting EBL procedure time.

rect placement of hemoclips on the vessel can be technically challenging because of a dome location or massive bleeding. Hemoclips can be placed indirectly in a zipper fashion in these challenging cases; however, complete hemostasis is often difficult to achieve [12].

Although EBL has been reported to be a safe and effective method [13-19], no studies on the feasibility of this technique have been published. The use of EBL is gradually increasing, but reinsertion of the colonoscope after the EBL device has been attached to its tip is considered cumbersome. In addition, the applicability and safety of EBL when performed by non-expert endoscopists have not been sufficiently verified. This study aimed to elucidate the feasibility of EBL when performed by non-expert endoscopists and the possibility of EBL as a standard method for treating colonic diverticular bleeding. 


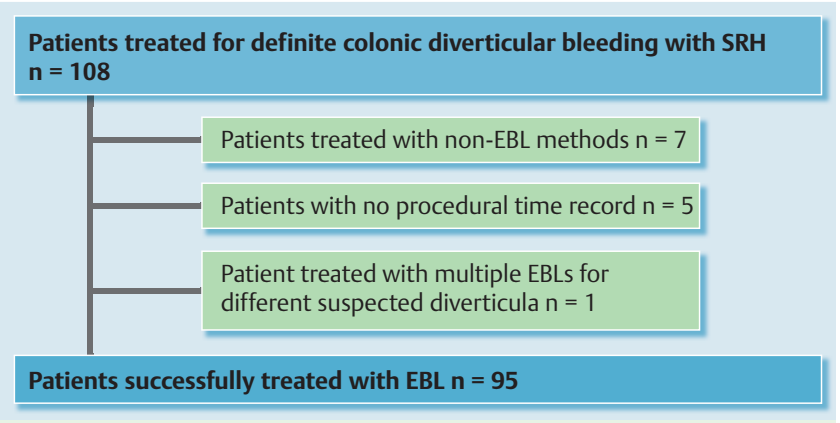

Fig. 1 Flow diagram for all patients included in a study of endoscopic band ligation for colonic diverticular bleeding performed by expert and non-expert endoscopists. SRH, stigmata of recent hemorrhage; EBL, endoscopic band ligation.

\section{Patients and methods}

$\nabla$

\section{Study population}

A retrospective cohort study was conducted at St. Luke's International Hospital, a tertiary referral center in Tokyo, Japan, between June 2009 and October 2014. A total of 108 patients with definite colonic diverticular bleeding and stigmata of recent hemorrhage (SRH), such as active bleeding, a nonbleeding visible vessel, and adherent clot [6], were treated during this period. Within the cohort, seven patients who had been treated with transcatheter arterial embolization, epinephrine injection, or endoscopic clipping were excluded. Epinephrine injection was used in six patients in whom the diverticula could not be adequately suctioned into the hood of the endoscopic ligator because of a small orifice. Two patients treated with epinephrine injection required additional transcatheter arterial embolization for persistent bleeding. In one patient with a diverticulum in the ascending colon, the orifice of the diverticulum with SRH was so large that the endoscope could be passed into the diverticulum. Therefore, endoscopic clipping was selected instead of EBL. Five patients were excluded because the procedure time was not recorded. One patient was also excluded because EBL was performed at two suspected sites of bleeding during the same intervention. A total of 95 patients who had successful initial hemostasis with EBL were analyzed in this study ( $\bullet$ Fig. 1).

\section{Endoscopic band ligation methods}

Bowel preparation with polyethylene glycol was performed before each examination to achieve a high rate of SRH identification. In patients who were hemodynamically unstable under intravenous fluid resuscitation, colonoscopy to identify the source of active bleeding was done without bowel preparation. Colonoscopy was performed with a water-jet scope (PCF-Q260AZI, PCFQ260JI, or GIF-Q260J; Olympus, Tokyo, Japan). When a diverticulum with SRH was identified, hemoclips (HX-610-135; Olympus) were applied as markers near the diverticulum ( $\bullet$ Fig. 2 a, - Fig. 2b). The endoscope was removed, and a band ligator device (MD-48710 EVL Device; Sumitomo Bakelite, Tokyo, Japan) was attached to its tip ( $\bullet$ Fig. 2c). The endoscope was then reinserted to the identified diverticulum. The diverticulum was aspirated into the transparent hood of the band ligator device, and an elastic O-ring was deployed ( $\bullet$ Fig.2d) [13-20]. Other endoscopic treatments, such as diluted epinephrine $(1: 20000)$ injection and endoscopic clipping, were considered for refractory bleeding when several EBL attempts by experts had been unsuccessful. Patients were followed on an outpatient basis at our institution for at least 30 days after EBL.

\section{Comparison of endoscopic band ligation treatments in the groups treated by expert and non-expert endoscopists}

Patients were classified into two groups: those treated by expert endoscopists and those treated by non-expert endoscopists. The expert endoscopists included institutional teaching staff of St. Luke's International Hospital who were also board-certified members of the Japanese Society of Gastrointestinal Endoscopy. The non-expert group included trainees who had completed training in routine colonoscopic procedures. None of the non-experts were board-certified members of the Japanese Society of Gastrointestinal Endoscopy, but they had performed more than 500 colonoscopies before performing EBL treatment. Because of the technical difficulties encountered in performing urgent colonoscopies, trainees in our institution are generally required to complete 500 colonoscopies before performing urgent colonoscopies without on-site assistance by highly experienced endoscopists. The decision regarding the selection of an operator (expert or non-expert) was left to the discretion of the staff physician. As previously stated, patients who were hemodynamically unstable with intravenous fluid resuscitation underwent colonoscopy without bowel preparation. Because colonoscopy in a patient without preparation may be difficult, with a low completion rate and impaired identification of SRH, these difficult cases may have been assigned to the experts. EBL procedure time (the time between marking the site of bleeding with hemoclips and completing the $\mathrm{O}$-band release) and total procedure time in these two groups were compared ( $\bullet$ Fig.3). Safety was determined based on EBL-associated adverse events.

\section{Statistical analysis}

For bivariate analyses, Student's $t$ test and Fisher's exact test were applied for continuous and categorical variables, respectively. If a non-normal distribution was suspected for a continuous variable, Wilcoxon's rank sum test was used.

Linear regression analyses were used to determine possible factors affecting EBL procedure time and total procedure time. A multivariate linear regression model was created by including independent variables with $P$ values of less than 0.2 in a simple linear regression model as well as including clinically important variables. All 95\% confidence intervals (CIs) were two-sided. $P$ values of less than 0.05 were considered significant. Statistical analyses were conducted with JMP version 9 (SAS Institute, Cary, North Carolina, USA). This study was approved by the ethics committee of St. Luke's International Hospital.

\section{Results \\ $\nabla$}

EBL achieved successful immediate hemostasis in all 95 patients in our study cohort (100\%). Six experts and six non-experts participated in the study. The median number of years of endoscopy experience was 14.0 (range 5-18) for the experts and 3.5 (range $1-7$ ) for the non-experts at the time of the EBL treatment. The mean (standard deviation [SD]) number of EBL cases performed by the experts during the study period was 8 (6), and the mean (SD) number performed by the non-experts was 8 (4).

The characteristics of the patients in the expert and non-expert groups are shown in Table 1. Experts performed 47 of the 95 


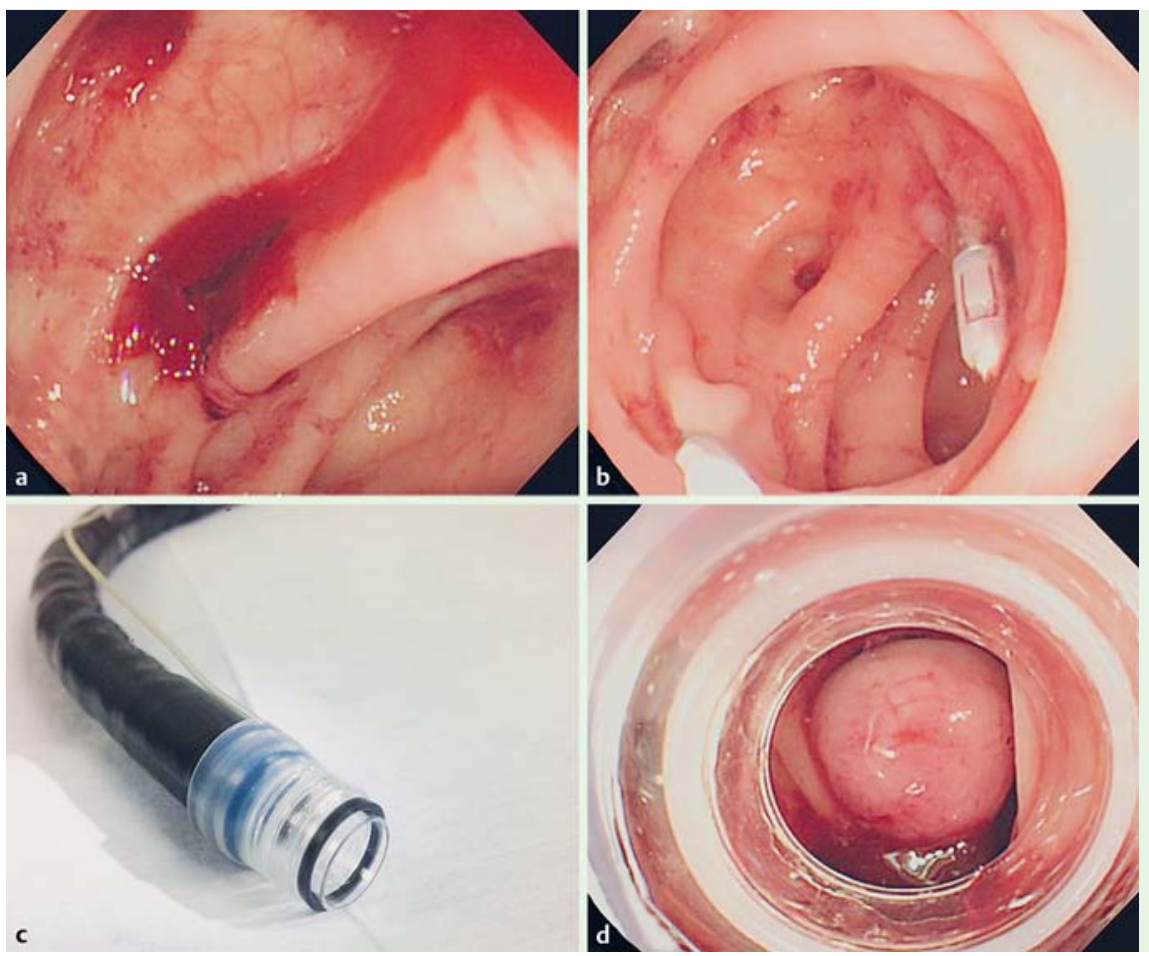

\begin{tabular}{|l|l|l|l|l|l|}
\hline & \\
1 & Time to identify the bleeding diverticulum (Fig. 2a) \\
2 & Marking with hemoclips near the diverticulum (Fig. 2b) \\
3 & Removal of the endoscope \\
4 & Attaching the band ligator device (Fig. 2c) \\
5 & Reinsertion to the diverticulum \\
6 & Endoscopic band ligation (Fig. 2d) \\
7 & Removal of the endoscope
\end{tabular}

Fig.2 a Endoscopic view of colonic diverticulum with active bleeding. $\mathbf{b}$ Marking with hemoclips near the diverticulum. $\mathbf{c}$ The colonoscope is removed, and a band ligator device is attached to its tip. d The colonoscope is reinserted, the diverticulum is aspirated into the transparent hood of the band ligator device, and the elastic O-band is deployed.

Fig. 3 Time frame of the endoscopic band ligation (EBL) procedure.

Table 1 Characteristics of the patients in a study of endoscopic band ligation (EBL) performed by expert and non-expert endoscopists.

\begin{tabular}{|c|c|c|c|}
\hline & Expert endoscopists $(n=47)$ & Non-expert endoscopists $(n=48)$ & $P$ value $^{1}$ \\
\hline Age, mean (SD), y & $66(14.2)$ & $65(14.1)$ & 0.70 \\
\hline Male, n (\%) & $32(68.0)$ & $40(83.3)$ & 0.10 \\
\hline Hypertension, n (\%) & $26(55.3)$ & $23(46.9)$ & 0.54 \\
\hline Anticoagulant, $\mathrm{n}(\%)$ & $18(38.3)$ & $13(27.1)$ & 0.28 \\
\hline Shock index, mean (SD) & $0.74(0.25)$ & $0.74(0.22)$ & 0.59 \\
\hline Hemoglobin, mean (SD), g/dL & $12.2(2.0)$ & $12.4(2.0)$ & 0.52 \\
\hline Right-sided diverticula, n (\%) & $37(78.7)$ & $36(75.0)$ & 0.81 \\
\hline Bowel preparation, $\mathrm{n}(\%)$ & $35(74.5)$ & $48(100)$ & 0.0001 \\
\hline Total procedure time, median (range), min & $33.0(19-101)$ & $33.5(13-72)$ & 0.63 \\
\hline EBL procedure time, median (range), min & $15.0(4-45)$ & $11.0(4-36)$ & 0.03 \\
\hline
\end{tabular}

SD, standard deviation.

1 Statistical significance was defined as $P<0.05$

procedures (49.5\%). Comorbidities, medications, shock index, hemoglobin level on admission, location of the bleeding diverticula, rate of bowel preparation, total procedure time, and EBL-associated adverse events were evaluated in each group. A significant difference was found between the rates of bowel preparation in the two groups, with all the patients treated by non-experts having undergone bowel preparation. The median EBL procedure time was 15 minutes (range 4-45) for the experts and $11 \mathrm{~min}$ utes (range $4-36)$ for the non-experts $(P=0.03)$. No significant 
Table 2 Simple and multiple linear regression models to investigate factors related to endoscopic band ligation procedure time.

\begin{tabular}{|c|c|c|c|c|c|c|}
\hline & \multicolumn{3}{|c|}{ Simple linear regression model } & \multicolumn{3}{|c|}{ Multiple linear regression model } \\
\hline & $\beta$ & $95 \% \mathrm{Cl}$ & $P$ value $^{1}$ & $\beta$ & $95 \% \mathrm{Cl}$ & $P$ value $^{1}$ \\
\hline Age & -0.02 & -0.12 to 0.08 & 0.68 & & & \\
\hline Male & -0.04 & -3.90 to 3.82 & 0.82 & & & \\
\hline Bowel preparation & -2.3 & -7.24 to 2.64 & 0.36 & 0.07 & -5.21 to 5.35 & 0.98 \\
\hline Right-sided diverticula & 3.9 & 0.10 to 7.70 & 0.045 & 3.75 & -0.09 to 7.59 & 0.05 \\
\hline Expert endoscopist & 3.1 & -0.10 to 6.30 & 0.06 & 2.95 & -0.53 to 6.43 & 0.09 \\
\hline
\end{tabular}

$\beta$, beta coefficient; $\mathrm{Cl}$, confidence interval.

1 Statistical significance was defined as $P<0.05$

Table 3 Simple and multiple linear regression models to investigate factors related to total procedure time.

\begin{tabular}{|c|c|c|c|c|c|c|}
\hline & \multicolumn{3}{|c|}{ Simple linear regression model } & \multicolumn{3}{|c|}{ Multiple linear regression model } \\
\hline & $\beta$ & $95 \% \mathrm{Cl}$ & $P$ value $^{1}$ & $\beta$ & $95 \% \mathrm{Cl}$ & $P$ value $^{1}$ \\
\hline Age & -0.01 & -0.12 to 0.08 & 0.91 & & & \\
\hline Male & -3.42 & -11.4 to 4.58 & 0.39 & & & \\
\hline Bowel preparation & -8.09 & -18.3 to 2.13 & 0.12 & -7.85 & -19.1 to 3.43 & 0.17 \\
\hline Right-sided diverticula & 1.89 & -6.25 to 10.0 & 0.64 & 1.06 & -7.14 to 9.26 & 0.80 \\
\hline Expert endoscopist & 2.14 & -4.72 to 9.00 & 0.53 & 0.10 & -7.32 to 7.43 & 0.98 \\
\hline
\end{tabular}

$\beta$, beta coefficient; $\mathrm{Cl}$, confidence interval.

${ }^{1}$ Statistical significance was defined as $P<0.05$

difference was found between the total procedure times in the two groups.

The results of a linear regression analysis to investigate factors related to EBL procedure time are shown in $\bullet$ Table 2. With use of a simple linear regression model, performance of EBL by experts and right-sided location of diverticula were factors that significantly increased EBL procedure time. In addition, multivariate analysis adjusted for the rate of bowel preparation, location of bleeding diverticula, and expertise in endoscopy revealed that a right-sided location of diverticula was the factor most significantly affecting EBL procedure time $(\beta=3.75 ; 95 \% \mathrm{CI}-0.09$ to 7.59; $P=0.05$ ).

The results of linear regression analysis to investigate factors related to total procedure time are reported in $\bullet$ Table 3 . Simple and multivariate linear regression models revealed no factors that significantly increased total procedure time.

Although all patients included in this study had successful initial hemostasis, 15 cases of rebleeding within 30 days occurred in this cohort. Five of these cases were in patients treated by nonexperts. In addition, in four cases more than one EBL attempt was required because of inadequate suctioning into the endoscopic ligator hood. Three of these patients requiring multiple EBL attempts were treated by experts, and one was treated by a non-expert. Two patients had right-sided diverticula and two had left-sided diverticula. Although up to four attempts were made by experts, one case with a right-sided diverticulum required 28 minutes for EBL completion. The median time with multiple EBL attempts was 21.5 minutes (range 6-28). There were no adverse events related to EBL in either group.

\section{Discussion}

$\nabla$

EBL achieves immediate hemostasis even in cases of massive bleeding. On the other hand, it requires reinsertion of the colonoscope after a band ligator device has been attached to its tip, which may be time-consuming. Furthermore, the endoscopic vis- ual field may become narrow by the attached band ligator device. For these reasons, EBL can be technically challenging compared with other hemostatic techniques.

As expected, a right-sided location of diverticula significantly lengthened the EBL procedure time because of the longer time required for reinsertion. Neither bowel preparation nor expertise in endoscopy affected EBL procedure time in multivariate analysis. Surprisingly, the expert endoscopists had longer EBL procedure times. It is unclear why the experts required more time to complete EBL procedures. However, one reason may be that experts were selected for the difficult cases with hemodynamic instability and considerable co-morbidities, thus prolonging the procedural time. As a result of technical improvements in EBL, non-experts are now able to learn EBL technique faster and more efficiently compared with the initial experiences of experts. In addition, in some cases experts supervised non-experts while they were performing procedures. At any rate, reinsertion of the colonoscope for EBL is fast and simple with less loop formation, so that advanced colonoscopy skills may not be required if the procedure is done in a patient with adequate bowel preparation. We believe that the EBL procedure can be performed with skills that are easily acquired.

We had 15 cases of rebleeding within 30 days in this cohort. In five of these cases, EBL was performed by a non-expert. With this limited number of patients, it is difficult to make assumptions about the cause of rebleeding; however, younger age, active bleeding of SRH, and left-sided lesions are considered risk factors for rebleeding [20]. In four cases, more than one attempt at banding was required because of inadequate suctioning into the endoscopic ligator hood. Even in these difficult cases, the median EBL procedure time was 21.5 minutes (range $6-28$ ). Therefore, repeated suctioning attempts may not substantially affect EBL procedure time. Moreover, only single-use band ligator devices were used in this study, whereas multiple-use band ligator devices may further decrease the time required for repeated attempts at EBL. Across our cohort, we did not experience any serious complications, such as perforation and infection. 
Some limitations of our study merit discussion. First, this was a retrospective study from a single institution, with a limited number of EBL cases performed by a limited number of endoscopists. Second, there may have been some selection bias regarding assignment of the endoscopists. Decisions regarding the selection of an operator (expert or non-expert) were left to the discretion of the staff physician and difficult cases may have been assigned to experts. In addition, although no change in endoscopist was made after the lesion to be treated with EBL had been identified, there may have been cases in which an expert took over to identify SRH, which may have affected the total procedure time. This is the first study to evaluate the possibility of standardizing the EBL procedure. Our findings suggest that the acquisition of technical skills for EBL is relatively straightforward and that the procedure can be completed in a short amount of time. As long as the endoscopists are well trained in standard colonoscopy and the patients have undergone bowel preparation, EBL is a feasible technique. We therefore conclude that within the limitations of our retrospective study, EBL can be safely and effectively performed by non-expert endoscopists.

\section{Competing interests: None}

\section{References}

1 Longstreth GF. Epidemiology and outcome of patients hospitalized with acute lower gastrointestinal hemorrhage: a population-based study. Am J Gastroenterol 1997; 92: 419-424

2 Chaudhry V, Hyser MJ, Gracias VH et al. Colonoscopy: the initial test for acute lower gastrointestinal bleeding. Am Surg 1998; 64: 723-728

3 Newman J, Fitzgerald JE, Gupta S et al. Outcome predictors in acute surgical admissions for lower gastrointestinal bleeding. Colorectal Dis 2012; 14: 1020-1026

4 McGuire HHJr. Bleeding colonic diverticula. A reappraisal of natural history and management. Ann Surg 1994; 220: 653-656

5 Poncet G, Heluwaert F, Voirin D et al. Natural history of acute colonic diverticular bleeding: a prospective study in 133 consecutive patients. Aliment Pharmacol Ther 2010; 32: 466-471
6 Jensen DM, Machicado GA, Jutabha R et al. Urgent colonoscopy for the diagnosis and treatment of severe diverticular hemorrhage. $\mathrm{N}$ Engl $\mathrm{J}$ Med 2000: 342; 78 - 82

7 Bloomfeld RS, Rockey DC, Shetzline MA. Endoscopic therapy of acute diverticular hemorrhage. Am J Gastroenterol 2001; 96: 2367-2372

8 Hokama A, Uehara T, Nakayoshi T et al. Utility of endoscopic hemoclipping for colonic diverticular bleeding. Am J Gastroenterol 1997; 92: $543-546$

9 Simpson PW, Nguyen MH, Lim JK et al. Use of endoclips in the treatment of massive colonic diverticular bleeding. Gastrointest Endosc 2004; 59: 433-437

10 Yen EF, Ladabaum U, Muthusamy VR et al. Colonoscopic treatment of acute diverticular hemorrhage using endoclips. Dig Dis Sci 2008; 53: $2480-2485$

11 Kaltenbach T, Watson R, Shah J et al. Colonoscopy with clipping is useful in the diagnosis and treatment of diverticular bleeding. Clin Gastroenterol Hepatol 2012; 10: 131-137

12 Ishii $\mathrm{N}$, Hirata $\mathrm{N}$, Omata $\mathrm{F}$ et al. Location in the ascending colon is a predictor of refractory colonic diverticular hemorrhage after endoscopic clipping. Gastrointest Endosc 2012; 76: 1175-1181

13 Witte JT. Band ligation for colonic bleeding: modification of multiband ligating devices for use with a colonoscope. Gastrointest Endosc 2000; 52: $762-765$

14 Farrell JJ, Graeme-Cook F, Kelsey PB. Treatment of bleeding colonic diverticula by endoscopic band ligation: an in-vivo and ex-vivo pilot study. Endoscopy 2003; 35: 823-829

15 Ishii $N$, Itoh $T$, Uemura $M$ et al. Endoscopic band ligation with a waterjet scope for the treatment of colonic diverticular hemorrhage. Dig Endosc 2010; 22: 232-235

16 Ishii $N$, Uemura $M$, Itoh $T$ et al. Endoscopic band ligation for the treatment of bleeding colonic and ileal diverticula. Endoscopy 2010; 42: $82-83$

17 Setoyama T, Ishii N, Fujita Y. Endoscopic band ligation (EBL) is superior to endoscopic clipping for the treatment of colonic diverticular hemorrhage. Surg Endosc 2011; 25: 3574-3578

18 Ishii N, Setoyama T, Deshpande GA et al. Endoscopic band ligation (EBL) for colonic diverticular hemorrhage. Gastrointest Endosc 2012; 75: $382-387$

19 Shibata S, Shigeno T, Fujimori K et al. Colonic diverticular hemorrhage: the hood method for detecting responsible diverticula and endoscopic band ligation for hemostasis. Endoscopy 2014; 46: 66-69

20 Ikeya T, Ishii $N$, Nakano $K$ et al. Risk factors for early rebleeding after endoscopic band ligation for colonic diverticular hemorrhage. Endosc Int Open 2015; 3: E523-E528 